\title{
A Blind Beam Tracking Scheme for Millimeter Wave Systems
}

\author{
Steve Blandino ${ }^{1,2}$, Thibault Bertrand ${ }^{3}$, Claude Desset ${ }^{2}$, André Bourdoux ${ }^{2}$, Sofie Pollin ${ }^{1,2}$, Jérôme Louveaux ${ }^{3}$ \\ ${ }^{1}$ Department of Electrical Engineering, KU Leuven, Belgium \\ ${ }^{2}$ imec, Belgium \\ ${ }^{3}$ Institute of Information and Communication Technologies, Electronics and Applied Mathematics, \\ UC Louvain, Belgium
}

\begin{abstract}
Millimeter-wave is one of the technologies powering the new generation of wireless communication systems. To compensate the high path-loss, millimeter-wave devices need to use highly directional antennas. Consequently, beam misalignment causes strong performance degradation reducing the link throughput or even provoking a complete outage. Conventional solutions, e.g. IEEE $802.11 \mathrm{ad}$, propose the usage of additional training sequences to track beam misalignment. These methods however introduce significant overhead especially in dynamic scenarios. In this paper we propose a beamforming scheme that can reduce this overhead. First, we propose an algorithm to design a codebook suitable for mobile scenarios. Secondly, we propose a blind beam tracking algorithm based on particle filter, which describes the angular position of the devices with a posterior density function constructed by particles. The proposed scheme reduces by more than $80 \%$ the overhead caused by additional training sequences.
\end{abstract}

Index Terms-Beam-tracking, mm-wave, beamforming, 802.11 ad, particle filter.

\section{INTRODUCTION}

Millimeter-wave (mm-wave) wireless communication is considered the enabling technology of next-generation wireless systems. More than $20 \mathrm{GHz}$ of spectrum is available at mmwave to accommodate the ever-increasing throughput requests thanks also to the advances on high-speed electronics enabling wireless systems operating at high carrier frequency and with wide modulation bandwidth.

The propagation path loss at mm-wave requires the use of large number of antennas to concentrate the radiated power into narrow beams. Consequently, the millimeter wave channel is sparse in space and beam misalignment causes strong signal to noise ratio (SNR) drops reducing the link throughput or even provoking a complete outage. Efficient beamforming strategies, which can correctly align the beams even in dynamic scenarios, are of crucial importance for mm-wave devices to guarantee ubiquitous coverage and the required throughput.

IEEE 802.11 ad/ay [1] provides explicit training sequences (TRN) appended at the end of data packets to support a beam tracking procedure (TRN-BT). These training sequences can be used to monitor the SNR and trigger a beam-sweep whenever a strong drop in SNR occurs. During the beam-sweep phase, both transmitter and receiver sequentially send TRN sequences on each antenna sector to find the steering providing the highest SNR. These procedures introduce latency, which leads to throughput reductions. Recently, algorithms predicting the device motion have been proposed to proactively adapt the beam pattern before an SNR drop occurs [2], [3].

Beyond the tracking schemes, the design of a robust codebook including wide beams, less sensitive to the device motion, can significantly reduce the training overhead at the cost of lower SNR. The analysis of a beamforming strategy, which include both code-book design and beam tracking for dynamic scenario is considered in [4], where a beamforming strategy was proposed by combining the codebook design with an anticipatory movement prediction algorithm that utilizes mobile devices sensors (accelerometer and magnetometer) to accurately forecast its next location.

In this paper, we present a beamforming strategy leveraging on both a wide beam and a blind motion prediction, reducing the triggering of beam-sweep events. The design of the proposed beamforming scheme provides two main contributions:

- Beam pattern design: we propose an algorithm to generate a code-book suitable for dynamic scenarios. The key idea of the proposed beam pattern is to enlarge the beamwitdh while minimizing the secondary lobes.

- Blind beam-tracking: we propose a predictive beamtracking algorithm based on particle filtering, which enables an accurate beamforming in dynamic scenarios, reducing by more than $80 \%$ the triggering of beam-sweep procedures.

The reminder of the paper is organized as follows: Section II presents the system model including the signal model, the frame structure and the channel model. Section III describes how to properly design the beam pattern in both static and dynamic scenarios. Section IV introduces the proposed blind beam-tracking algorithm. Section $\mathrm{V}$ presents the performance of the proposed beamforming scheme. Finally, Section VI gives the concluding remarks.

\section{SySTEM MODEL}

In this section, we present the system model used in the paper. We describe the structure of the considered mm-wave communication system including signal model, frame structure and channel model. 


\section{Channel Model}

Given the sparse nature of the mm-wave channel in time and space a quasi-deterministic channel model [6] is used

Fig. 1. 802.11 ad frame structure. TRN field is an optional overhead to enable beam-tracking.

\section{A. Signal Model}

Consider a mm-wave system comprising a fixed access point (AP) with $M$ antennas and one moving user station (STA) with $N$ antennas. Assume that the antennas, in both devices, are organized as uniform linear array (ULA) composed of isotropic radiators each provided with a phase shifter. While in this paper we focus on the design of the beam for a single user system, these schemes are valid for the analog beamforming design of hybrid MIMO architectures usually used in multiuser scenarios [5].

Assume a wideband single carrier system (SC-FDE) as in $802.11 \mathrm{ad} / \mathrm{ay}$. The circulant property satisfied by using the cyclic prefix permits to describe the downlink transmission as follow:

$$
y[k]=\mathbf{f}_{\mathrm{STA}}^{\mathrm{H}} \mathbf{H}[k] \mathbf{f}_{\mathrm{AP}} x[k]+n,
$$

where $x[k]$ is the equivalent transmitted symbol at the subcarrier $k, \mathbf{f}_{\mathrm{AP}}$ and $\mathbf{f}_{\mathrm{STA}}$ are the $\mathbb{C}^{M \times 1}$ and the $\mathbb{C}^{N \times 1}$ the antenna weight vectors (AWV), i.e. frequency flat analog beamformer and combiner vectors of the AP and STA respectively. $\mathbf{H}$ is the $\mathbb{C}^{N \times M}$ frequency selective channel. $n$ is the additive Gaussian noise with variance $\sigma_{n}$.

\section{B. Frame structure}

AP and the STA communicate using two different frame structures:

1) Beam training frames contain pilot sequences, e.g. Golay sequences, which are used in the first phase of the communication when the communication link is not established or when an outage occurs and the link needs to be restored. The initial access procedure is successful when $\mathbf{f}_{\mathrm{AP}}$ and $\mathbf{f}_{\mathrm{STA}}$ are acquired.

2) Data frames, in Figure 1, are used when the link is established. At the beginning of the data frame, shorter pilot sequences compared to the beam training frames (STF, $\mathrm{CEF}$ ) are present to provide synchronization, SNR and channel estimation or CFO and phase noise correction. Optional training overhead (TRN) can be appended at the end of a packet to allow beam training procedures.

A finite set of vectors is usually available since the transceivers need to quickly switch between the different vectors, hence predefined vectors are generally stored in a codebook to reduce computation complexity and power consumption. In the following we propose a robust code-book for dynamic scenarios from which $\mathbf{f}_{\mathrm{AP}}$ and $\mathbf{f}_{\mathrm{STA}}$ are selected. Devices motion however makes the initial $\mathbf{f}_{\mathrm{AP}}$ and $\mathbf{f}_{\mathrm{STA}}$ outdated. Our beam-tracking algorithm uses the mandatory overhead (STF and $\mathrm{CEF}$ ), without adding the additional overhead at the end of the packet (TRN) to update $\mathbf{f}_{\mathrm{AP}}$ and $\mathbf{f}_{\mathrm{STA}}$. to represent realistic multi-path components. The design of the beam-tracking schemes presented in this paper relies on the line-of sight (LOS) component of the channel, since at mm-wave only the LOS component can provide reliably high transmission rate. The description of the normalized $N \times M$ LOS channel is given by:

$$
\overline{\mathbf{H}}=\sqrt{M N} \alpha \mathbf{a}_{r}\left(\phi_{r}\right) \mathbf{a}_{t}\left(\phi_{t}\right)^{H},
$$

where $\alpha$ is the complex gain of the channel at the distance $d$ and $\phi_{t}$ and $\phi_{r}$ are the azimuth angles of departure and arrival respectively, which depend on the relative position of the devices. The vectors $\mathbf{a}_{r}$ and $\mathbf{a}_{t}$ are the normalized receive and transmit array steering vectors respectively, which incorporate all of the spatial characteristics of the array. For an $N$-elements uniform linear array oriented along the $y$-axis, the array steering vector $\mathbf{a}$ is:

$$
\mathbf{a}=\left[1 e^{j 2 \pi \frac{d}{\lambda} \sin \phi} e^{j 2 \pi \frac{2 d}{\lambda} \sin \phi} \cdots e^{j 2 \pi \frac{(N-1) d}{\lambda} \sin \phi}\right]^{T},
$$

where $\lambda$ is the carrier wavelength $d$ is the inter-element spacing. We omit the description of the elevation since an ULA has omni-directional pattern in the elevation dimension.

\section{BEAM CODEBOOK DESIGN}

Large multi-antenna transceivers focus their radiations to a narrow region, hence the beam should be accurately tuned to exploit the beamforming gain. In this section we describe how to properly design a code-book to maximize the SNR in both static and dynamic scenarios.

\section{A. Beam Codebook for Static Devices}

In the case of static devices the design of narrow pencil beams optimizing the SNR is desired. This is possible by adapting the phase coefficients $\mathbf{f}_{\mathrm{STA}}$ and $\mathbf{f}_{\mathrm{AP}}$ such that:

$$
\begin{aligned}
& \left(\mathbf{f}_{\mathrm{STA}}^{\mathrm{opt}}, \mathbf{f}_{\mathrm{AP}}^{\mathrm{opt}}\right)=\underset{\mathbf{f}_{\mathrm{AP}}, \mathbf{f}_{\mathrm{STA}}}{\arg \max } \quad\left|\mathbf{f}_{\mathrm{STA}}^{H} \overline{\mathbf{H}} \mathbf{f}_{\mathrm{AP}}\right|^{2}, \\
& \text { subject to } \mathbf{f}_{\mathrm{AP}} \in \mathcal{F}_{\mathrm{AP}}, \quad\left\|\mathbf{f}_{\mathrm{AP}}\right\|=1 \\
& \mathbf{f}_{\mathrm{STA}} \in \mathcal{F}_{\mathrm{STA}}, \quad\left\|\mathbf{f}_{\mathrm{STA}}\right\|=1
\end{aligned}
$$

where $\mathcal{F}_{\mathrm{AP}}$ and $\mathcal{F}_{\mathrm{STA}}$ are the feasible set of the AP and STA beamsteering vectors.

With full channel knowledge a simple solution is based on singular value decomposition. Letting the channel's singular value decomposition be $\overline{\mathbf{H}}=\mathbf{U} \boldsymbol{\Sigma} \mathbf{V}$, the optimal vectors are $\mathbf{f}_{\mathrm{AP}}^{\mathrm{opt}}=\mathbf{U}^{(1)}$ and $\mathbf{f}_{\mathrm{STA}}^{\mathrm{opt}}=\mathbf{V}^{(1)}$, the right and left eigenvector associated with the strongest eigenvalue. However since the sparse nature of the mm-wave channel, the optimal SVD beamforming vectors are given by the array steering vector in the strongest direction [7] that is:

$$
\begin{aligned}
\mathbf{f}_{\mathrm{STA}} & =\mathbf{a}_{t}\left(\phi_{t}\right), \\
\mathbf{f}_{\mathrm{AP}} & =\mathbf{a}_{r}\left(\phi_{r}\right)^{H},
\end{aligned}
$$

hence the estimation of $\phi_{t}$ and $\phi_{r}$ are sufficient to solve the problem in (4). 


\section{B. Beam Codebook for Dynamic Devices}

For a large number of antennas, in the case of a scenario with moving STAs the approach presented in $[1 \mathrm{II}$-A may not be desirable since a small mismatch between the estimation and the true value of the angle directions can cause a drastic SNR drop.

To guarantee a more reliable link even with a large number of antennas, we modify problem (4) such that the SNR is not maximized only for the combination $\left(\phi_{t}, \phi_{r}\right)$ but it is maximized for the set of angles $\left(\Phi_{t}, \Phi_{r}\right)$, in which $\Phi_{t}=\left[\phi_{t}-\right.$ $\left.\Delta \phi_{\mathrm{bw}} / 2, \phi_{t}+\Delta \phi_{\mathrm{bw}} / 2\right]$ and $\Phi_{r}=\left[\phi_{r}-\Delta \phi_{\mathrm{bw}} / 2, \phi_{r}+\right.$ $\left.\Delta \phi_{\mathrm{bw}} / 2\right]$, with $\Delta \phi_{\mathrm{bw}}$ indicating the beam-width. In this case the optimization problem is given by:

$$
\begin{aligned}
\left(\mathbf{f}_{\mathrm{STA}}^{\text {opt }}, \mathbf{f}_{\mathrm{AP}}^{\text {opt }}\right)=\underset{\mathbf{f}_{\mathrm{AP}}, \mathbf{f}_{\mathrm{STA}}}{\arg \max } & \left|\mathbf{f}_{\mathrm{STA}}^{H} \overline{\mathbf{H}} \mathbf{f}_{\mathrm{AP}}\right|^{2}, \\
\text { subject to } & \mathbf{f}_{\mathrm{AP}} \in \mathcal{F}_{\mathrm{AP}}, \quad\left\|\mathbf{f}_{\mathrm{AP}}\right\|=1 \\
& \mathbf{f}_{\mathrm{STA}} \in \mathcal{F}_{\mathrm{STA}}, \quad\left\|\mathbf{f}_{\mathrm{STA}}\right\|=1 \\
& \Delta \phi_{\mathrm{bw}}=C .
\end{aligned}
$$

where $C$ is a constant.

The problem is solved separately for the AP and the STA, e.g. the codebook of the AP is designed assuming an omnidirectional receiver STA. A solution of the problem is provided by using a conventional evolutionary algorithm. The main idea is to translate problem (6) into a fitness function. The AWV with higher fitness in the population will have higher chances of survival. At each iteration a new generation of AWV is introduced by modifying the AWV of the previous generation. After few generations, the selected AWV is likely to have high fitness values and therefore represent a (sub-)optimal solution. To solve this problem, we minimize the following fitness function $\mathbb{F}_{\mathrm{AP}}$ :

$$
\begin{aligned}
& \mathbb{F}_{\mathrm{AP}}\left(\mathbf{f}_{\mathrm{AP}}\right)=\underbrace{\frac{1}{\Delta \phi_{\mathrm{bw}}} \int_{\phi \in \Phi_{t}}\left(A_{m}-|A(\phi)|\right)^{2} d \phi}_{\mathbb{F}_{1}\left(\mathbf{f}_{\mathrm{AP}}\right)} \\
& \beta_{1} \underbrace{\frac{1}{\pi-\Delta \phi_{\mathrm{bw}}} \int_{\phi \in \bar{\Phi}}|A(\phi)|^{2} d \phi}_{\mathbb{F}_{2}\left(\mathbf{f}_{\mathrm{AP}}\right)}- \\
& \beta_{2} \underbrace{\frac{1}{\Delta \phi_{\mathrm{bw}}} \int_{\phi \in \Phi_{t}}(|A(\phi)|)^{2} d \phi}_{\mathbb{F}_{3}\left(\mathbf{f}_{\mathrm{AP}}\right)}
\end{aligned}
$$

where $\bar{\Phi}=\left[-\pi / 2, \phi_{t}-\Delta \phi_{\mathrm{bw}} / 2\right] \cup\left[\phi_{t}+\Delta \phi_{\mathrm{bw}} / 2, \pi / 2\right]$ is the region outside the beamwidth, $A(\phi)=\mathbf{a}_{t} \mathbf{f}_{\mathrm{AP}}^{H}, A_{m}=$ $\mathbb{E}\{|A(\phi)|\}$ averaged in $\Phi_{t} . \beta_{1}$ and $\beta_{2}$ are arbitrary real scalars used for weighting $\mathbb{F}_{1}\left(\mathbf{f}_{\mathrm{AP}}\right), \mathbb{F}_{2}\left(\mathbf{f}_{\mathrm{AP}}\right)$ and $\mathbb{F}_{3}\left(\mathbf{f}_{\mathrm{AP}}\right)$. The fitness function in equation (7) refers to the codebook design of the AP, however a similar function can be written for the STA. The fitness function has been chosen to minimize the array gain in $\bar{\Phi}\left(=\mathbb{F}_{2}\left(\mathbf{f}_{\mathrm{AP}}\right)\right)$, minimize the level of ripples of $|A(\phi)|$ in $\Phi$ $\left(=\mathbb{F}_{1}\left(\mathbf{f}_{\mathrm{AP}}\right)\right)$ and maximize the array gain in $\Phi\left(=\mathbb{F}_{3}\left(\mathbf{f}_{\mathrm{AP}}\right)\right)$.

Algorithm 1 can be summarized as follow. In the first stage a random population of $\mathrm{AWV} \mathbf{F}_{\mathrm{AP}} \in \mathbb{C}^{N \times N_{P}}$, where $N_{P}$ is the size of the population, is generated. The coefficients

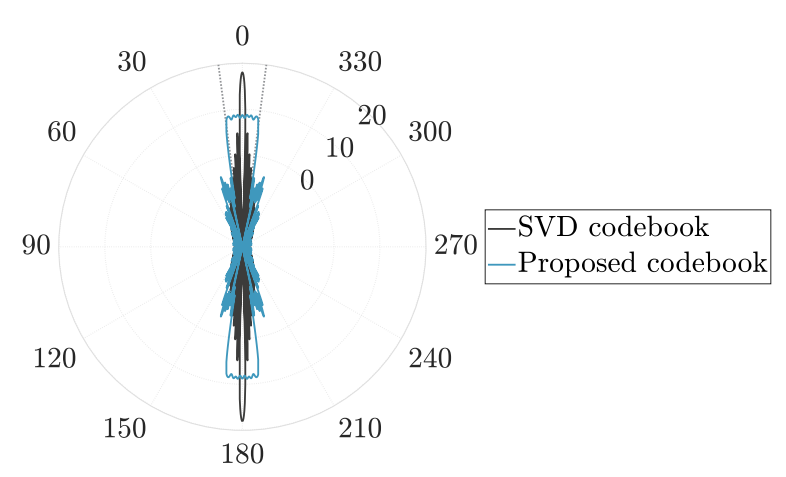

Fig. 2. Array pattern for $\phi_{t}=0$ for both SVD and proposed codebooks assuming 64 antennas. The proposed codebook enlarge the beamwidth such that $\Delta \phi_{\mathrm{bw}}=15^{\circ}$. Array gain is expressed in $\mathrm{dB}$.

of $\mathbf{F}_{\mathrm{AP}}$ have unitary amplitude but a random phase. At each iteration, the best individual (in the sense of the minimisation of $\mathbb{F}\left(\mathbf{f}_{\mathrm{AP}}\right)$ ) is selected and a new population is created from the perturbation of this individual.

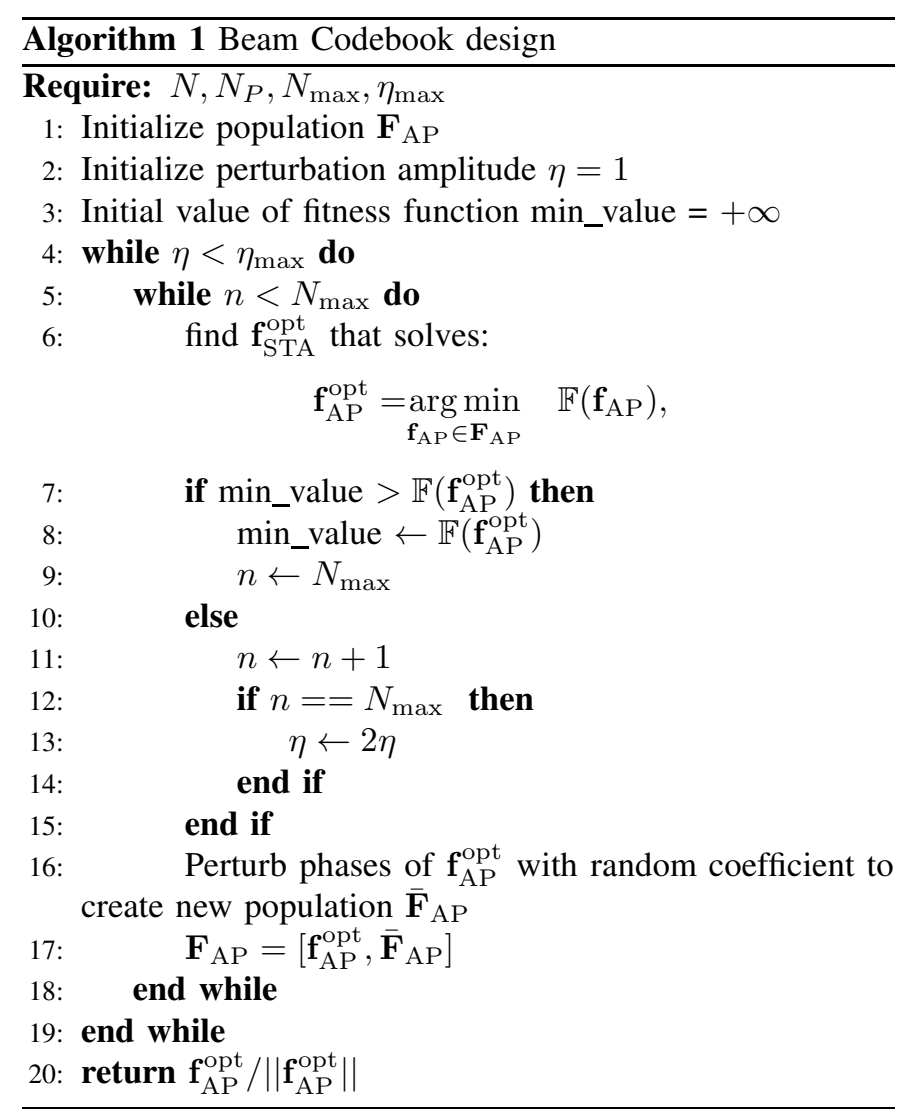

Figure 2 shows an example of beam pattern when the AP uses 64 antennas for $\phi_{t}=0$ generated by designing the codebook according with algorithm 1. A larger beam-width is obtained compared to a conventional SVD codebook to prevent SNR drops in dynamic scenarios. 


\section{BEAM TRACKING SCHEMES}

The SNR of the link usually decreases over time due to the devices mobility. When the SNR becomes too low, generally below a threshold, it becomes necessary to find a more suitable AWV. Instead of performing an exhaustive beam search using additional TRN sequences, predictive beam-tracking schemes that can anticipate the device motion, can be used. In this section we first review the conventional beam-tracking used in 802.11 ad systems. Then, we propose a novel beam tracking scheme based on particle filtering.

\section{A. Training Sequence Beam Tracking (TRN-BT)}

A procedure named beam tracking has been included in the IEEE 802.11ad specification and allows a fast beam refinement. This beam tracking procedure is a request/response procedure. Transmitter training (TRN-T) and receiver training (TRN-R) fields are appended at the end of data packets so that the STAs can train their transmit and receive AWVs. During the transmission of a TRN-R field, the same transmit AWV must be used for the transmission of all TRN subfields so that the receiver can train its receive AWV. Inversely, during the reception of a TRN-T field, the same receive AWV must be used so that the transmitter can train its transmit AWV.

\section{B. Predict and track with Particle Filter (PF-BT)}

To estimate the user motion, and predict the best AWV without sending TRN sequnces, we propose a tracking scheme based on particle filter (PF). The PF can estimate the past, current or future states of a Markov process using noisy and partial observations.

Consider the Markov process:

$$
\begin{aligned}
\theta_{t} & =\theta_{t-1}+\dot{\theta}_{t-1} \Delta t \\
\dot{\theta}_{t} & =f\left(\dot{\theta}_{t-1} ; \nu_{t}\right) \\
\gamma_{t} & =g\left(\theta_{t} ; w_{t}\right)
\end{aligned}
$$

where $\theta_{t}$ is the angular position and $\dot{\theta}_{t}$ is the angular velocity of the user every $\Delta t$ ms. $\theta_{t}$ and $\dot{\theta}_{t}$ represent the state $\boldsymbol{\Theta}_{t}$ of the Markov process, while $\gamma_{t}$ is the observed SNR. $f$ and $g$ are functions describing the process evolution. $\nu_{t}$ and $w_{t}$ are random variables. We would like to estimate recursively the posterior distribution of the hidden state $p\left(\boldsymbol{\Theta}_{t} \mid \gamma_{1: t-1}\right)$ using a PF. The problem can be addressed recursively in two steps [8]:

- Prediction:

$$
\begin{aligned}
p\left(\boldsymbol{\Theta}_{t} \mid \gamma_{1: t-1}\right) & =\int p\left(\boldsymbol{\Theta}_{t}, \boldsymbol{\Theta}_{t-1} \mid \gamma_{1: t-1}\right) d \boldsymbol{\Theta}_{t-1} \\
& =\int p\left(\boldsymbol{\Theta}_{t} \mid \boldsymbol{\Theta}_{t-1}\right) p\left(\boldsymbol{\Theta}_{t-1} \mid \gamma_{1: t-1}\right) d \boldsymbol{\Theta}_{t-1}
\end{aligned}
$$

- Update:

$$
\begin{aligned}
p\left(\boldsymbol{\Theta}_{t} \mid \gamma_{1: t}\right) & =\frac{\left.p\left(\gamma_{t} \mid \boldsymbol{\Theta}_{t}\right) p\left(\boldsymbol{\Theta}_{t}\right) \mid \gamma_{1: t-1}\right)}{p\left(\gamma_{t} \mid \gamma_{1: t-1}\right)} \\
& =\frac{p\left(\gamma_{t} \mid \boldsymbol{\Theta}_{t}\right) p\left(\boldsymbol{\Theta}_{t} \mid \gamma_{1: t-1}\right)}{\int p\left(\gamma_{t} \mid \boldsymbol{\Theta}_{t}\right) p\left(\boldsymbol{\Theta}_{t} \mid \gamma_{1: t-1}\right) d \boldsymbol{\Theta}_{t}} .
\end{aligned}
$$

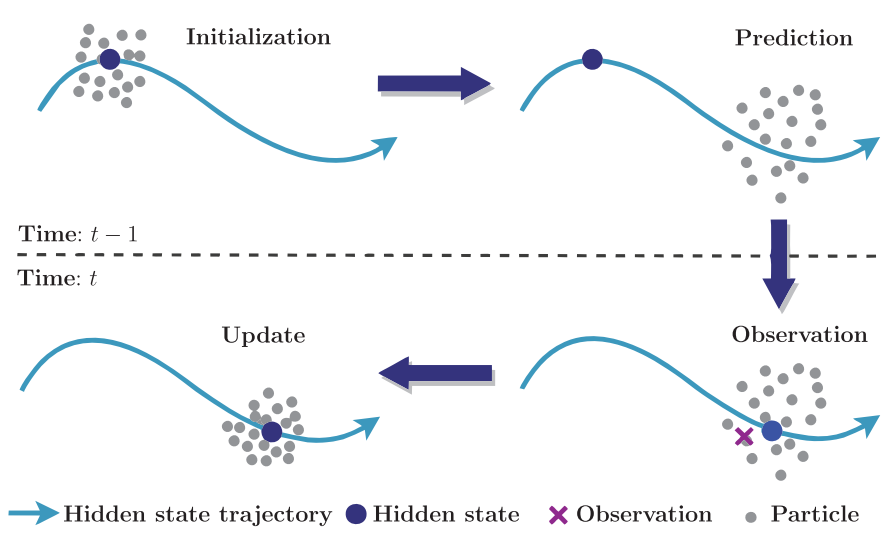

Fig. 3. Illustration of the particle filter.

The PF represents the probabilistic distribution of the hidden state by particles. Each particle can be seen as one possible evolution of the hidden state and the density of the particles describes the probabilistic distribution of the hidden state. A given hidden state is more likely if many particles have a state close to it. The particle filter operations are illustrated in Figure 3 At each iterations, the prediction and update steps are performed. The prediction step uses the probabilistic distribution of the hidden state $p\left(\boldsymbol{\Theta}_{t-1} \mid \gamma_{1: t-1}\right)$ and based on the conditional probabilistic state evolution $p\left(\boldsymbol{\Theta}_{t} \mid \boldsymbol{\Theta}_{t-1}\right)$ estimates the most probable state at the next time instant applying the function $f_{t}\left(\boldsymbol{\Theta}_{t-1}, \nu_{t}\right)$ to each particle. The update step estimates the most probable state given all the observations up to time $t$ by using the predicted distribution $p\left(\boldsymbol{\Theta}_{t} \mid \gamma_{1: t-1}\right)$ and the conditional probabilistic state observation $p\left(\gamma_{t} \mid \boldsymbol{\Theta}_{t}\right)$. Particles are selected according to $p\left(\gamma_{t} \mid \boldsymbol{\Theta}_{t}\right)$ and are then resampled. A resampling operation ensures that a sufficient number of particles can describe the probabilistic distribution of the hidden state.

The evolution of the angular speed $\dot{\theta}_{t}$ of the user needs to consider two different scenarios:

- The user motion at time $t$ follows the one at time $t-1: f\left(\dot{\theta}_{t-1} ; \nu_{t}\right)=\dot{\theta}_{t-1}+\nu_{t}^{1}$. In this case, $\nu_{t}^{1}$ can be considered as a random Gaussian variable expressing the angular acceleration of the user.

- The user motion completely change between time $t-1$ and time $t: f\left(\dot{\theta}_{t-1} ; \nu_{t}\right)=\nu_{t}^{2}$. In this case, $\nu_{t}^{2}$ can be considered as a random variable taken from a uniform distribution.

The two scenarios can have different probability of occurrence, hence during the prediction step, two unequal sets of particles can be considered such that each set is updated with a different distribution.

\section{Simulation}

Let us consider an indoor scenario where a single moving STA is communicating with a stationary AP. We assume that the antenna array of the STA is made of a single antenna element, without losses of generalities, and we test our proposed schemes at the AP. The AP is provided with an antenna 


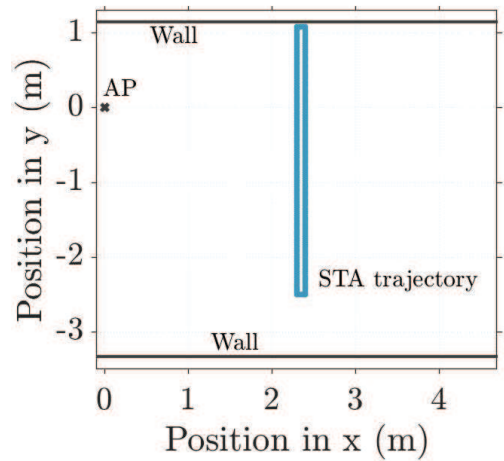

Fig. 4. Indoor scenario with a fixed AP and a moving STA.

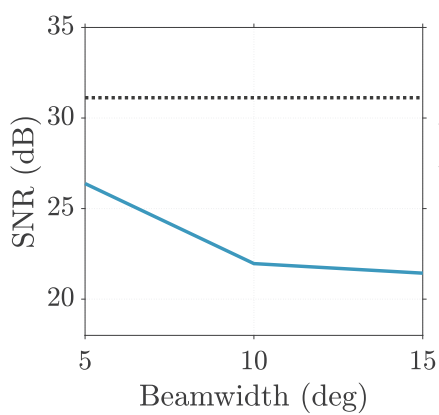

(a) Static

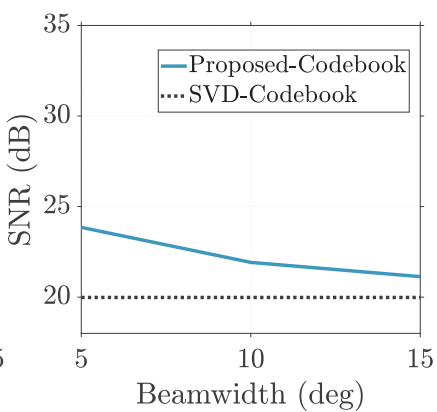

(b) Dynamic
Fig. 5. SNR vs beamwidth in static and dynamic scenarios.

array of $M=64$ antennas. Let us also consider that the STA can move with a speed of $5 \mathrm{~km} / \mathrm{h}=1.39 \mathrm{~m} / \mathrm{s}$ and follows the trajectory depicted in Figure 4 . It is assumed that the AP knows perfectly the initial angular position of the STA and thus the beam is correctly steered toward the STA.

First, we test our codebook design in both static and dynamic scenarios. We show the results for $\beta=2, N_{P}=100, N_{\max }=200$ and $\eta_{\max }=10^{5}$. Figure 5 shows the simulated average SNR, when the STA follow the trajectory making 2 complete loops. The SNR is presented varying $\Delta \phi_{\mathrm{bw}}$, the beamwidth of our designed codebook according to Algorithm 1. The figure shows also the SNR achieved assuming a conventional SVD based codebook. In dynamic scenarios the design of a wider beam allows to achieve higher average SNR with an improvement of $4 \mathrm{~dB}$ compared to a traditional SVD based coodebook when $\Delta \phi_{\text {bw }}=5^{\circ}$. As expected instead in a static scenario the SVD codebook achieves the optimal performance. These results suggest that future $\mathrm{mm}$-wave devices need to use codebooks, which include both very directional beams to maximize the SNR in static scenario, but also wider beams are needed to guarantee high throughput even in mobile scenarios.

Second, we analyze the performance of the proposed predictive beam tracking. We assume that sudden motion changes are

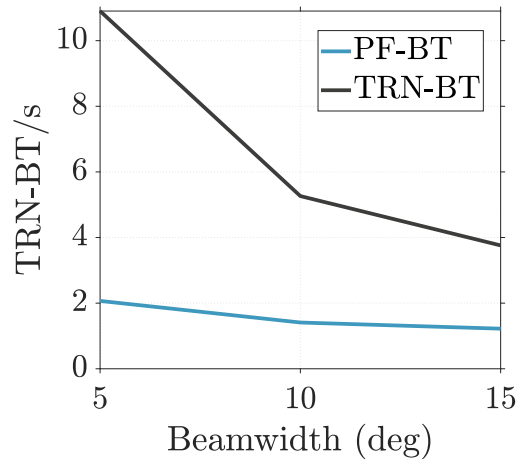

Fig. 6. Frequency of TRN-BT procedure against beamwidth with and without PF-BT.

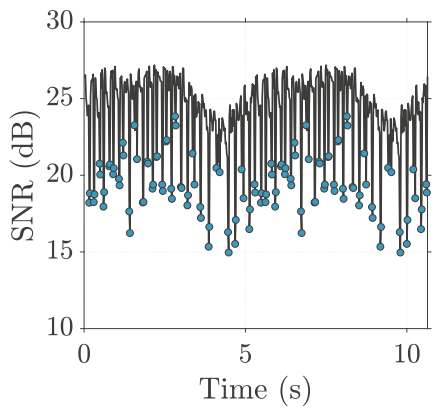

(a) TRN-BT

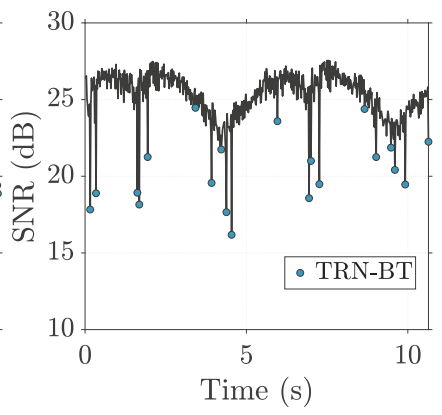

(b) PF-BT
Fig. 7. SNR temporal evolution with and without PF-BT

less likely than a continuous movement, hence we assume that $98 \%$ of the particles update their angular velocity accordingly with $\nu_{t}^{1}$ while the rest with $\nu_{t}^{2}$. The choice of the distribution $\nu_{t}^{1}$ and $\nu_{t}^{2}$ can be related with the maximal angular speed of the user. We assume the angular speed of the user to be smaller than $\dot{\theta}_{\max }$. Considering a user moving with an absolute speed inferior to $1.5 \mathrm{~m} / \mathrm{s}$, standing constantly at more than $1 \mathrm{~m}$ from the AP, the maximal angular speed of the user is $\dot{\theta}_{\max }=\operatorname{atan}\left(\frac{1.5}{1}\right) \approx 56^{\circ} / s . \nu_{t}^{1}$ can be described as a random variable taken from a zero mean Gaussian distribution of standard deviation $\dot{\theta}_{\max } \Delta t$ meaning that about $68 \%$ of the particles will accelerate by less than $56^{\circ} / s^{2}$ between $t-1$ and $t . \nu_{t}^{2}$ can be described as a random variable taken from a uniform distribution in the range $\left[-\dot{\theta}_{\max } ; \dot{\theta}_{\max }\right]$. When an SNR drop is experienced a TRN-BT is performed. The AP can transmit TRN training sequences every $20 \mathrm{~ms}$ to test different AWVs. Figure 6 shows the number of TRN-BT/s, that is the frequency of triggering a beam sweep procedure by varying the codebook beam-width. When the AP performs particle filtering for tracking the received SNR and predicting the user motion, the frequency of a TRN-BT procedure is reduced by more than $80 \%$. By increasing the codebook beam-width the number of TRN-BT/s reduces since the user stays longer inside the beam.

Finally, Figure 7 shows the temporal evolution of the SNR for a user in motion. When beam-tracking relies only on TRN, 
the SNR drops continuously. When the AP predict the motion of the STA using PF-BT, the SNR experienced by the STA is much more stable with few interruptions.

\section{CONCLUSION}

This paper proposes a beamforming procedure, which leverages on two main contributions. First, we propose an algorithm to design a beam codebook that offers a larger coverage compared to conventional solutions. The proposed codebook is particularly relevant in dynamic scenarios in which an improvement of $4 \mathrm{~dB}$ in the average SNR is achieved. By increasing the codebook beam-width the user stays longer inside the main lobe experiencing less detrimental SNR drops.

Second, we propose a beam tracking algorithm based on particle filtering. PF effectively predicts the user movement only with SNR measurements. Hence, PF can reduce more than $80 \%$ the triggering of overhead pilots to re-align the beams.

As a future work, a study considering different user motions should be done to highlight the robustness of the proposed algorithms. Moreover, experimental validations would be interesting to test the robustness of the proposed scheme with hardware non-idealities and a real propagation channel. Moreover, this scheme reduce the peak gain, hence another interesting question is the maximum achievable range and the highest modulation order achievable by the system.

\section{REFERENCES}

[1] IEEE 802.11 working group, "IEEE 802.11ad, amendment 3: Enhancements for very high throughput in the $60 \mathrm{GHz}$ band," December 2012.

[2] J. Kang, I. Orikumhi, Y.-M. Park, and S. Kim, "A millimeter wave beam tracking in vehicular scenario via particle filter," in 2018 International Conference on Network Infrastructure and Digital Content (IC-NIDC). IEEE, 2018, pp. 234-238

[3] C. Zhang, D. Guo, and P. Fan, "Tracking angles of departure and arrival in a mobile millimeter wave channel," in 2016 IEEE International Conference on Communications (ICC), May 2016, pp. 1-6.

[4] Z. Yang, P. H. Pathak, Y. Zeng, and P. Mohapatra, "Sensor-assisted codebook-based beamforming for mobility management in $60 \mathrm{GHz}$ WLANs," in 2015 IEEE 12th International Conference on Mobile Ad Hoc and Sensor Systems, Oct 2015, pp. 333-341.

[5] S. Blandino, G. Mangraviti, C. Desset, A. Bourdoux, P. Wambacq, and S. Pollin, "Multi-user hybrid MIMO at $60 \mathrm{GHz}$ using 16-antenna transmitters," IEEE Transactions on Circuits and Systems I: Regular Papers, vol. 66, no. 2, pp. 848-858, Feb 2019.

[6] R. J. Weiler, M. Peter, W. Keusgen, A. Maltsev, I. Karls, A. Pudeyev, I. Bolotin, I. Siaud, and A.-M. Ulmer-Moll, "Quasi-deterministic millimeter-wave channel models in MiWEBA," EURASIP Journal on Wireless Communications and Networking, Mar 2016. [Online]. Available: https://doi.org/10.1186/s13638-016-0568-6

[7] O. E. Ayach, R. W. Heath, S. Abu-Surra, S. Rajagopal, and Z. Pi, "The capacity optimality of beam steering in large millimeter wave mimo systems," in 2012 IEEE 13th International Workshop on Signal Processing Advances in Wireless Communications (SPAWC), June 2012, pp. $100-104$.

[8] A. Doucet, N. de Freitas, and N. Gordon, "An introduction to sequential monte carlo methods," Sequential Monte Carlo Methods in Practice. Springer, Berlin, 012001. 